\title{
New-onset cough and fever in a toddler with stage 5 chronic kidney disease: Questions
}

\author{
Cassandra A. Petri ${ }^{1}$. Yalile Perez ${ }^{2}$. Leah S. Heidenreich ${ }^{1} \cdot$ Carl H. Cramer $^{3} \cdot$ Timothy M. Olson $^{2} \cdot$ Nathan C. Hull $^{4}$. \\ Christian Hanna ${ }^{3}$ (1)
}

Received: 28 August 2021 / Revised: 30 August 2021 / Accepted: 31 August 2021 / Published online: 11 October 2021

(c) The Author(s), under exclusive licence to International Pediatric Nephrology Association 2021

A 2-year-old female was admitted to the hospital after presenting to the emergency department with a 3-day history of dry cough, one episode of emesis, and low-grade fever. Her medical history was significant for systemic hypertension and polyuric, non-dialysis-dependent stage 5 chronic kidney disease (CKD) related to branchio-oto-renal (BOR) dysplasia syndrome, for which she was active on the deceased donor waiting list for kidney transplant.

Upon initial presentation, she was thin and well-appearing with normal blood pressure and heart rate and increased respiratory rate. Her oxygen saturation was normal in room air. There was no evidence of edema or weight gain from baseline. The cardiovascular and respiratory examinations were remarkable for mild tachypnea without retractions and the presence of a pericardial friction rub.

Initial blood studies showed an elevated CRP of $61.7 \mathrm{mg} / \mathrm{L}$ (normal $<8.0$ ), mildly low albumin at $3.3 \mathrm{~g} /$
$\mathrm{dL}$ (normal 3.5-5), a normal white blood count of $11.7 \times 10^{9} / \mathrm{L}$ (normal 4.9-13.2), a low hemoglobin of $7.7 \mathrm{~g} / \mathrm{dL}$ (normal 10.2-12.7), and an elevated platelet count of $570 \times 10^{9} / \mathrm{L}$ (normal 189-394). Blood chemistry panel showed a high serum creatinine of $7.54 \mathrm{mg} / \mathrm{dL}$ (normal 0.19-0.49), high blood urea nitrogen (BUN) of $58 \mathrm{mg} / \mathrm{dL}$ (normal 7-22), low total calcium at $7.3 \mathrm{mg} /$ $\mathrm{dL}$ (normal 9.3-10.6), and low potassium at $3.0 \mathrm{mEq} / \mathrm{L}$ (normal 3.6-5.2). A catheterized urine sample demonstrated more than $300 \mathrm{mg} / \mathrm{dL}$ protein with a specific gravity of 1.020 but was otherwise unremarkable, and urine culture was negative. Respiratory pathogen panel and SARS-CoV-2 PCR tests collected by nasopharyngeal swab were negative. A chest radiograph demonstrated new enlargement of the cardiac silhouette compared to the study obtained 1 month prior to presentation (Fig. 1a, b).

The answers to these questions can be found at https://doi.org/10. 1007/s00467-021-05301-7.

Christian Hanna

Hanna.Christian@mayo.edu

1 Department of Pediatric and Adolescent Medicine, Mayo Clinic, Rochester, MN, USA

2 Division of Pediatric Cardiology, Department of Pediatric and Adolescent Medicine, Mayo Clinic, Rochester, MN, USA

3 Division of Pediatric Nephrology and Hypertension, Department of Pediatric and Adolescent Medicine, Mayo Clinic, Rochester, MN, USA

4 Division of Pediatric Radiology, Mayo Clinic, Rochester, MN, USA 

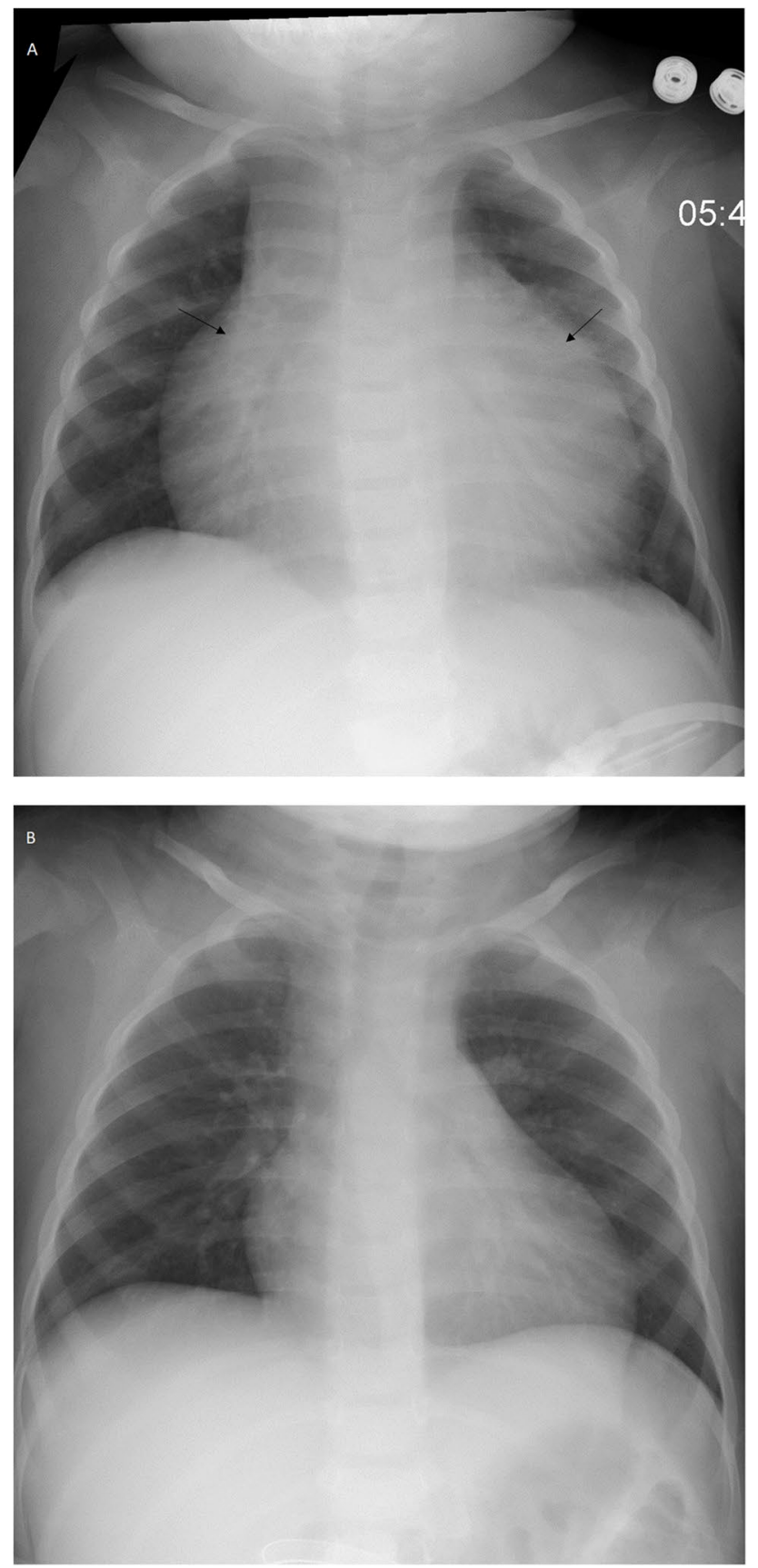

Fig. 1 Chest radiograph showing markedly enlarged cardiac silhouette (arrows) (a) compared to the radiograph obtained 1 month prior to admission (b). Lungs are without pulmonary edema, focal consolidation, or pleural effusion

\section{Questions}

1. What would be your differential diagnosis for an enlarged cardiac silhouette in a toddler with advanced CKD?

2. What additional investigations would you perform?

3. How would you treat this patient?

Data availability Not applicable.

Code availability Not applicable.

\section{Declarations}

Conflict of interest The authors declare no competing interests.

Publisher's note Springer Nature remains neutral with regard to jurisdictional claims in published maps and institutional affiliations. 